\title{
Imipenem/Cilastatin/Relebactam: A Review in Gram-Negative Bacterial Infections
}

\author{
Young-A. Heo ${ }^{1}$
}

Accepted: 18 January 2021 / Published online: 25 February 2021

(c) Springer Nature Switzerland AG 2021

\begin{abstract}
Imipenem/cilastatin/relebactam (Recarbrio ${ }^{\mathrm{TM}}$ ) is an intravenously administered combination of the carbapenem imipenem, the renal dehydropeptidase-I inhibitor cilastatin, and the novel $\beta$-lactamase inhibitor relebactam. Relebactam is a potent inhibitor of class $A$ and class $C \beta$-lactamases, conferring imipenem activity against many imipenem-nonsusceptible strains. Imipenem/cilastatin/relebactam is approved in the USA and EU for the treatment of hospital-acquired bacterial pneumonia (HABP) and ventilator-associated bacterial pneumonia (VABP) in adults and other gram-negative infections, including complicated urinary tract infections (cUTIs) [including pyelonephritis] and complicated intra-abdominal infections (cIAIs), in adults with limited or no alternative treatment options. In pivotal phase II and III trials, imipenem/cilastatin/relebactam was noninferior to piperacillin/tazobactam in patients with HABP/VABP and to imipenem/cilastatin in patients with cUTIs and cIAIs. It was also effective in imipenem-nonsusceptible infections. Imipenem/cilastatin/relebactam was generally well tolerated, with a safety profile consistent with that of imipenem/cilastatin. Available evidence indicates that imipenem/cilastatin/ relebactam is an effective and generally well tolerated option for gram-negative infections in adults, including critically ill and/or high-risk patients, and a potential therapy for infections caused by carbapenem-resistant pathogens.
\end{abstract}

\section{Introduction}

An increasing prevalence of multidrug resistant (MDR) gram-negative pathogens, which frequently involves the production of $\beta$-lactamases, is of significant global concern [1]. Carbapenems are broad-spectrum $\beta$-lactam antibacterial agents that play a critical role in the management of complicated and serious infections caused by such pathogens, especially against strains producing

Digital Features for this Adis Drug Evaluation can be found at https ://doi.org/10.6084/m9.figshare.13322738.

The manuscript was reviewed by: $C$. F . Carpenter, Oakland University William Beaumont School of Medicine, Rochester, MN, USA; $\boldsymbol{P}$. Cristofaro, Infectious Disease Division, Brown Medical School, Providence, RI, USA; J. L. Kuti, Center for AntiInfective Research and Development, Hartford Hospital, Hartford, CT, USA; $\boldsymbol{C}$. N. Yost, Infectious Diseases, Beaumont Hospital, Royal Oak, MN, USA; R. Zaragoza, Intensive Care Department, Hospital Universitario Dr. Peset, Valencia, Spain.

Young-A. Heo

demail@springer.com

1 Springer Nature, Private Bag 65901, Mairangi Bay, Auckland 0754, New Zealand
Imipenem/cilastatin/relebactam: clinical considerations in gram-negative infections

Potent in vitro activity against Enterobacterales and Pseudomonas aeruginosa isolates, including carbapenem-nonsusceptible strains expressing class $\mathrm{A}$ and/or $\mathrm{C}$ $\beta$-lactamases

Effective in adults with HABP/VABP, cUTIs or cIAIs, including infections caused by imipenem-nonsusceptible pathogens

Generally well tolerated

extended-spectrum $\beta$-lactamases (ESBLs) [2]. However, the growing emergence and spread of carbapenemresistant pathogens worldwide exacerbates the clinical challenge of treating these infections [3-5]. Polymyxins (i.e. colistin and polymyxin B), tigecycline and aminoglycosides have been commonly used to treat carbapenemresistant infections but these therapies are often associated with low efficacy (due to resistance, pharmacokinetic and pharmacodynamic profiles) and high toxicity [6-8]. Consequently, there is an urgent need for the development of 
new antibacterial agents with improved clinical outcomes and safety profiles against such infections.

Resistance mechanisms to carbapenems include upregulation of antimicrobial efflux pumps, porin loss and the production of $\beta$-lactamase enzymes that hydrolyze carbapenems, such as Klebsiella pneumoniae carbapenemases (KPCs) [8-10]. One strategy to overcome $\beta$-lactamasemediated resistance and to restore the antibacterial activity of carbapenems is to combine them with suitable $\beta$-lactamase inhibitors [8-10].

Imipenem/cilastatin/relebactam (Recarbrio ${ }^{\mathrm{TM}}$ ) is an intravenously administered combination of the carbapenem imipenem, the renal dehydropeptidase-I inhibitor cilastatin, and the novel $\beta$-lactamase inhibitor relebactam $[11,12]$. Imipenem/cilastatin/relebactam is approved in the USA [11] and EU [12] for the treatment of adults with hospital-acquired bacterial pneumonia (HABP) and ventilator-associated bacterial pneumonia (VABP), and other gram-negative infections, including complicated urinary tract infections (cUTIs) [including pyelonephritis] and complicated intra-abdominal infections (cIAIs), in adults with limited or no alternative treatment options (Sect. 6) $[11,12]$.

This article reviews the therapeutic efficacy, safety and tolerability of imipenem/cilastatin/relebactam, with its pharmacological properties also summarized.

\section{Pharmacodynamic Properties}

\subsection{Chemistry and Mechanism of Action}

Imipenem is a carbapenem that, like other $\beta$-lactam antibacterials, inhibits cross-linking of peptidoglycan during cell wall synthesis by inactivating penicillin binding proteins, ultimately leading to bacterial cell lysis and death [2]. Imipenem is coadministered with cilastatin, a dehydropeptidase-I inhibitor, to reduce its renal metabolism; cilastatin does not have any antibacterial activity [2].

Relebactam is a novel $\beta$-lactamase inhibitor of class $A$ and $C \beta$-lactamases [2,9]. Although relebactam has no intrinsic antibacterial activity, it can protect imipenem from degradation by Ambler class A (e.g. KPCs) and class C (e.g. AmpC) $\beta$-lactamases and Pseudomonas-derived cephalosporinase (PDC) [14-15]; however, relebactam is not active against class B metallo- $\beta$-lactamases (MBLs) [e.g. NDM, VIM and IMP] or class D oxacillinases (e.g. OXA-48) [2, 9]. In vitro, the addition of relebactam significantly improves the antibacterial activity of imipenem by lowering the minimum inhibitory concentration (MIC) of imipenem by 2- to 128-fold against ESBL- or KPC-producing Enterobacterales, as well as MDR or imipenem-resistant Pseudomonas aeruginosa isolates [2, 10, 16-20]. Furthermore, neither imipenem nor relebactam are subject to efflux, which can be an advantage against strains that overexpress efflux pumps [20].

\subsection{In Vitro Activity}

This section focuses on the in vitro antibacterial activity of imipenem/relebactam against clinically relevant isolates of gram-negative bacteria associated with approved indications for this drug combination, including HABP/VABP, cUTI and cIAI $[11,12]$. In the US prescribing information [11] and/or EU summary of product characteristics [12], specified aerobic gram-negative pathogens include Acinetobacter baumannii [11, 12], Enterobacter species (including E. cloacae [11, 12] and E. asburiae [12]), Escherichia coli $[11,12]$, Klebsiella species (including K. aerogenes, K. oxytoca, K. pneumoniae [11, 12]), Serratia marcescens $[11,12]$, Citrobacter species (including $C$. freundii [11, 12] and C. koseri [12]) and P. aeruginosa [11, 12].

Data are primarily drawn from the ongoing Study for Monitoring Antimicrobial Resistance Trends (SMART) surveillance program, in which clinical isolates have been collected in the USA [21-23], Europe [24], China [25] or worldwide [26-28]. Data reported in this section are for isolates collected between 2015 and 2018. In SMART, isolates are collected from bloodstream, lower respiratory tract, intra-abdominal and/or urinary tract infections. Isolates are tested for susceptibility to imipenem/relebactam (with relebactam at a fixed concentration of $4 \mathrm{mg} / \mathrm{L}$ ) and a range of comparators using Clinical and Laboratory Standards Institute (CLSI) broth microdilution methodology and interpreted using European Committee on Antimicrobial Susceptibility Testing (EUCAST) or CLSI breakpoints [21-28]. Approved EUCAST breakpoints for imipenem/ relebactam against Enterobacterales and P. aeruginosa are $\leq 2 \mu \mathrm{g} / \mathrm{mL}$ for susceptibility and $>2 \mu \mathrm{g} / \mathrm{mL}$ for resistance [29]. Approved CLSI breakpoints for imipenem/relebactam are: Enterobacterales (susceptible $\leq 1 \mu \mathrm{g} / \mathrm{mL}$, intermediate susceptible $2 \mu \mathrm{g} / \mathrm{mL}$ and resistant $\geq 4 \mu \mathrm{g} / \mathrm{mL}$ ) and $P$. aeruginosa ( $\leq 2,4$ and $\geq 8 \mu \mathrm{g} / \mathrm{mL}$, respectively) [30]. Prior to EUCAST and CLSI breakpoints for imipenem/ relebactam being approved, the existing imipenem breakpoints were used to interpret the analyses [21-24, 26-28].

Imipenem/relebactam exhibited potent in vitro activity against all Enterobacterales isolates collected between 2015 and 2018, with the susceptibility rate being $\approx 4-45 \%$ higher than that of most other tested comparators (Table 1) [22-26]. The susceptibility rates of imipenem/relebactam were $>90 \%$ against seven of the ten most commonly found Enterobacterales species collected worldwide as part of the SMART 2017 surveillance program, which were $E$. coli $(n$ 
$=14,194 ; 99.6 \%$ susceptible $), K$. pneumoniae $(n=7280$; $93.0 \%)$, E. cloacae $(n=1609 ; 96.8 \%), K$. oxytoca $(n=1013$; $99.4 \%), K$. aerogenes $(n=831 ; 97.6 \%), C$. freundii $(n=$ $568 ; 98.9 \%)$ and $C$. koseri $(n=403 ; 99.8 \%)$ [26]. Imipenem/ relebactam demonstrated modest activity against $S$. marcescens $(n=1133 ; 70.6 \%)$ and weak activity against Morganella morganii $(n=538 ; 32.0 \%)$, with relebactam increasing the susceptibility rates to imipenem by $18.6 \%$ and $26.4 \%$, respectively. Addition of relebactam did not increase the activity of imipenem against Proteus mirabillis $(n=1687)$, with the susceptibility rate for imipenem/relebactam and imipenem alone being $63.0 \%$ and $63.7 \%$, respectively [26]. It should be noted that members of Morganellaceae (e.g. Morganella species, Proteus species and Providencia species) are intrinsically less susceptible or resistant to imipenem by a mechanism independent of $\beta$-lactamase production [25]. Against A. baumannii isolates collected in the USA in the SMART 2016-2018 surveillance program $(n=156)$, imipenem/relebactam demonstrated limited benefit relative to imipenem alone (48.7\% vs $47.4 \%$ ), as many strains of this pathogen harbour class D oxacillinases [21].

Imipenem/relebactam maintained potent in vitro activity against subsets of Enterobacterales isolates with specific $\beta$-lactam-nonsusceptible or MDR phenotypes (Table 1) [22-25]. The addition of relebactam to imipenem restored imipenem susceptibility to $42.4-66.3 \%$ of imipenem-nonsusceptible isolates and increased susceptibility rates to imipenem by $\approx 4-11 \%$ for cefepime-nonsusceptible, ceftazidimenonsusceptible, piperacillin/tazobactam-nonsusceptible and MDR isolates (Table 1). Of the other tested comparators, only amikacin and colistin exhibited comparable activities to those of imipenem/relebactam [22, 24]. Imipenem/ relebactam also exhibited excellent in vitro activity against KPC-producing Enterobacterales, with the susceptibility rate being $\approx 20$ to $>90 \%$ higher than all other tested comparators (Table 1) [24, 26]. In addition, in vitro activity of

\section{Table 1 In vitro activity of imipenem/relebactam against selected clinical isolates}

\begin{tabular}{|c|c|c|c|c|c|c|c|c|}
\hline \multirow[t]{2}{*}{ Pathogen } & \multirow{2}{*}{$\begin{array}{l}\text { No. } \\
\text { of isolates }\end{array}$} & \multicolumn{7}{|c|}{$\%$ susceptible $\left(\mathrm{MIC}_{90}[\mu \mathrm{g} / \mathrm{mL}]\right)$} \\
\hline & & IMI/REL & IMI & $\mathrm{PIP} / \mathrm{TZP}$ & FEP & AMK & $\mathrm{CEF}$ & COL \\
\hline $\begin{array}{l}\text { All Entero- } \\
\text { bacterales } \\
{[22-26]}\end{array}$ & 71,345 & $\begin{array}{l}93.1-99.1 \\
\text { (1) }\end{array}$ & $\begin{array}{l}86.7-97.4 \\
(8)\end{array}$ & $\begin{array}{c}79.3-90.6 \\
(>64)\end{array}$ & $\begin{array}{c}50.4-89.7 \\
\quad(>32)\end{array}$ & $\begin{array}{l}74.4-99.6 \\
(16)\end{array}$ & $\begin{array}{r}60-86.7 \\
(>32)\end{array}$ & $\begin{array}{l}84.3-95.7 \\
(2)\end{array}$ \\
\hline $\begin{array}{l}\text { CEF-NS } \\
{[22,24]}\end{array}$ & 4993 & $\begin{array}{l}94.1-98.7 \\
(0.5)\end{array}$ & $\begin{array}{l}90.2-90.5 \\
\text { (1) }\end{array}$ & $\begin{array}{c}39.8-48.7 \\
(>64)\end{array}$ & $\begin{array}{c}19.1-34.1 \\
(>32)\end{array}$ & $\begin{array}{l}87.8-98.0 \\
(8)\end{array}$ & $0(>32)$ & $\begin{array}{r}92.5-93 \\
4(\leq 1)\end{array}$ \\
\hline $\begin{array}{l}\text { FEP-NS } \\
{[22,24]}\end{array}$ & 4334 & $\begin{array}{l}93.4-98.4 \\
(0.25)\end{array}$ & $\begin{array}{l}89.2-89.8 \\
\text { (2) }\end{array}$ & $\begin{array}{c}40.2-60.6 \\
(>64)\end{array}$ & $0(>32)$ & $\begin{array}{l}86.0-97.3 \\
(16)\end{array}$ & $\begin{array}{c}7.6-17.6 \\
(>32)\end{array}$ & $\begin{array}{r}94.0-96 \\
1(\leq 1)\end{array}$ \\
\hline $\begin{array}{l}\text { IMI-NS }^{\mathrm{a}} \\
{[24,25]}\end{array}$ & 1639 & $\begin{array}{c}42.4-66.3 \\
(>32)\end{array}$ & $0(>32)$ & $\begin{array}{c}6.3-20.9 \\
(>64)\end{array}$ & $\begin{array}{c}9.3-15.4 \\
(>32)\end{array}$ & $\begin{array}{c}50.9-54 \\
(>32)\end{array}$ & $\begin{array}{l}6.5-16 \\
(>32)\end{array}$ & $\begin{array}{r}67.1-83 . \\
6(>4)\end{array}$ \\
\hline $\begin{array}{c}\text { PIP/TZP-NS } \\
{[22,24]}\end{array}$ & 3944 & $\begin{array}{l}92.5-98.1 \\
(0.5)\end{array}$ & $\begin{array}{l}86.8-87.8 \\
(4)\end{array}$ & $0(>64)$ & $\begin{array}{c}34.5-55.2 \\
(>32)\end{array}$ & $\begin{array}{l}85.2-97.5 \\
(8)\end{array}$ & $\begin{array}{l}24.6-27.0 \\
(>32)\end{array}$ & $\begin{array}{r}91.3-91 \\
9(\leq 1)\end{array}$ \\
\hline $\begin{array}{l}\text { MDR } \\
{[22-24]}\end{array}$ & 6519 & $\begin{array}{l}92.1-98.2 \\
(0.5)\end{array}$ & $\begin{array}{l}82.3-89.8 \\
\text { (2) }\end{array}$ & $\begin{array}{c}36.7-50.6 \\
(>64)\end{array}$ & $\begin{array}{r}13-39.0 \\
(>32)\end{array}$ & $\begin{array}{l}86.7-97.8 \\
(8)\end{array}$ & $\begin{array}{c}4.8-23.6 \\
(>32)\end{array}$ & $\begin{array}{r}84.8-92 . \\
2(\leq 1)\end{array}$ \\
\hline $\begin{array}{c}\text { KPC-positive } \\
{[24,26]}\end{array}$ & 563 & $\begin{array}{l}94.6-98.6 \\
\text { (1) }\end{array}$ & $\begin{array}{c}1.4-3.1 \\
(>32)\end{array}$ & $\begin{array}{l}0-0.5 \\
\quad>64)\end{array}$ & $\begin{array}{r}1.4-4.0 \\
(>32)\end{array}$ & $\begin{array}{c}34.8-70.6 \\
(>32)\end{array}$ & $\begin{array}{l}0.7-5.2 \\
\quad(>32)\end{array}$ & $\begin{array}{r}73.2-73 . \\
9(>4)\end{array}$ \\
\hline $\begin{array}{c}\text { Pseudomonas } \\
\text { aeruginosa } \\
{[23,27]}\end{array}$ & 14,902 & $\begin{array}{l}90.8-93.9 \\
(2)\end{array}$ & $\begin{array}{l}69.0-72.0 \\
(16)\end{array}$ & $\begin{array}{c}69.1-70.2 \\
\quad(>64)\end{array}$ & $\begin{array}{c}75.0-75.6 \\
(32)\end{array}$ & $\begin{array}{l}91.1-96.0 \\
(16)\end{array}$ & $\begin{array}{c}74.3-76.9 \\
\quad>32)\end{array}$ & $\begin{array}{r}99.0-99 \\
6(\leq 1)\end{array}$ \\
\hline CEF-NS [27] & 3132 & $69.6(>32)$ & $34.4(>32)$ & $8.6(>64)$ & $17.7(>32)$ & $72.6(>32)$ & $0(>32)$ & $98.2(2)$ \\
\hline FEP-NS [27] & 3047 & $67.2(>32)$ & $30.4(>32)$ & $10.3(>64)$ & $0(>32)$ & $69.9(>32)$ & $15.4(>32)$ & $98.2(2)$ \\
\hline IMI-NS [27] & 3776 & $70.3(>32)$ & $0(>32)$ & $36.8(>64)$ & $43.9(>32)$ & $76.0(>32)$ & $45.6(>32)$ & $98.4(2)$ \\
\hline $\begin{array}{l}\text { PIP/TZP-NS } \\
{[27]}\end{array}$ & 3760 & $73.1(>32)$ & $36.5(>32)$ & $0(>64)$ & $27.3(>32)$ & $76.9(>32)$ & $23.8(>32)$ & $98.6(\leq 1)$ \\
\hline $\begin{array}{l}\text { MDR } \\
{[23,27]}\end{array}$ & 4594 & $\begin{array}{l}70.7-82.2 \\
(>32)\end{array}$ & $\begin{array}{l}28.8-38.9 \\
(>32)\end{array}$ & $\begin{array}{c}10.0-15.8 \\
(>64)\end{array}$ & $\begin{array}{c}21.1-29.6 \\
(>32)\end{array}$ & $\begin{array}{c}72.9-89.8 \\
(>32)\end{array}$ & $\begin{array}{l}20.3-32.4 \\
(>32)\end{array}$ & $\begin{array}{l}97.9-99.0 \\
(2)\end{array}$ \\
\hline
\end{tabular}

Pathogens against which imipenem/cilastatin/relebactam has demonstrated efficacy in SMART surveillance program [22-27]. Clinical isolates were collected in the EU (2015-2017 [24]), USA (2015-2017 [23], 2016 [22]), China (2015-2018 [25]) or worldwide (2015-2016 [27], 2017 [26]). Not all studies reported $\mathrm{MIC}_{90}$. Some data are available only as abstracts/posters [26]

AMK amikacin, CEF ceftazidime, COL colistin, FEP cefepime, KPC Klebsiella pneumoniae carbapenemase, IMI imipenem, IMI/REL imipenem/relebactam, $M I C_{90}$ minimum inhibitory concentration required to inhibit $90 \%$ of isolates, $M D R$ multidrug resistant, $N S$ nonsusceptible, PIP/TZP piperacillin/ tazobactam

${ }^{a}$ Including isolates carrying metallo- $\beta$-lactamases and/or OXA-48-like carbapenemases 
imipenem/relebactam against KPC-producing carbapenemresistant Enterobacterales was generally comparable to that of ceftazidime/avibactam [31].

Imipenem/relebactam also demonstrated potent in vitro activity against $P$. aeruginosa (Table 1 ) [23, 27]. Of the tested comparators, only amikacin and colistin demonstrated comparable activities that approximated or exceeded those of imipenem/relebactam, with the susceptibility rates to other tested comparators being 15-25\% lower than that of imipenem/relebactam. Similarly, the susceptibility rates of imipenem/relebactam against the subsets of $P$. aeruginosa isolates with specific $\beta$-lactam-nonsusceptible and MDR phenotypes were $25-60 \%$ higher than those of tested comparators, except amikacin and colistin [23, 27]. In particular, the addition of relebactam to imipenem increased imipenem susceptibility of imipenem-nonsusceptible $P$. aeruginosa isolates to $70.3 \%$ [27]. Moreover, the in vitro susceptibility profile of imipenem/relebactam against carbapenem-nonsusceptible $P$. aeruginosa isolates was generally similar to that of ceftazidime/avibactam [8], with imipenem/relebactam retaining high activity against $P$. aeruginosa isolates that had developed resistance to ceftolozane/tazobactam and ceftazidime/avibactam [32].

Overall, the MIC values required to inhibit the growth of $90 \%$ of isolates $\left(\mathrm{MIC}_{90}\right)$ for imipenem/relebactam against Enterobacterales and $P$. aeruginosa isolates (including those that were susceptible to imipenem [20]) were 2- to $\geq 32$-fold lower than those for imipenem alone (Table 1) [22-27]. Furthermore, relative to imipenem alone, imipenem/relebactam $\mathrm{MIC}_{90}$ values were two- to fourfold lower against ESBL- and AmpC-producing Enterobacterales isolates $(n=5428$ and 984, respectively) collected globally in the SMART 2016 surveillance program [33]. The susceptibility rates to imipenem/relebactam were generally similar across the various included infection sources [21, 22, 24-28].

\subsection{In Vivo Activity}

The in vitro activity of imipenem/relebactam is supported by evidence from animal models of infections due to imipenem-resistant bacterial strains, including murine models of disseminated infection [34], neutropenic thigh infection [35-37] or pulmonary infection [34, 38].

\subsection{Pharmacodynamic/Pharmacokinetic Considerations}

As established with other $\beta$-lactams, the best predictor of the antibacterial efficacy of imipenem is the percentage of the dosing interval that the unbound plasma concentration of imipenem exceeds the imipenem MIC ( $\% \mathrm{f}$ T $>$ MIC) [11, 12]. For carbapenems, $\% f \mathrm{~T}>\mathrm{MIC}$ of $20 \%$ and $30-40 \%$ is required to achieve bacterial stasis and $1-$ to $2-\log _{10}$ kill, respectively $[33,39]$. In an in vitro hollow-fibre infection model, the clinically approved dose of imipenem $500 \mathrm{mg}$ plus relebactam $250 \mathrm{mg}$ maintained imipenem $\% f \mathrm{~T}>$ MIC well above the required level for bactericidal activity (i.e. $>40 \%$ ) against Enterobacterales and $P$. aeruginosa isolates [39]. Based on this study, the pharmacokinetic/pharmacodynamic (PK/PD) target for imipenem combined with relebac$\operatorname{tam} \% f \mathrm{~T}>\mathrm{MIC}$ was established as $6.5 \%[39,40]$.

The best predictor of relebactam efficacy is the ratio of the $24 \mathrm{~h}$ unbound relebactam area under the concentration time curve (AUC) to imipenem MIC (fAUC/MIC), whereby imipenem was in the presence of relebactam $4 \mu \mathrm{g} / \mathrm{mL}[11$, 12], according to data from in vivo neutropenic mouse thigh infection [35, 36] and neutropenic mouse lung infection [38] models, as well as in vitro checkerboard and hollow-fibre studies [41]. For example, in the neutropenic mouse thigh infection model, the magnitude of relebactam $24 \mathrm{~h} f \mathrm{AUC} /$ MIC required for bacterial stasis, $1-$ and $2-\log _{10}$ kill of $P$. aeruginosa strains was 3.3, 4.3 and 7.0, respectively [36]. In addition, in a translational model based on checkerboard and hollow-fibre studies, a relebactam $24 \mathrm{~h} f$ AUC/MIC of 7.5 was associated with a $2-\log _{10}$ kill of imipenem-resistant $P$. aeruginosa strains, suggesting robust, conservative and comparable 2-log kill PK/PD targets for relebactam across various preclinical models [41]. The PK/PD target for relebactam $24 \mathrm{~h} f$ AUC/MIC was established as $\geq 5.2[35,40]$.

Based on the imipenem \% $f \mathrm{~T}>\mathrm{MIC}$ and relebactam $24 \mathrm{~h}$ $f$ AUC/MIC targets corresponding to a $2-\log _{10}$ kill, a population pharmacokinetic model in patients with different renal function categories (including augmented renal clearance) predicted that the approved dosing regimens of imipenem/ cilastatin/relebactam (Sect. 6) were sufficient to reach $>90 \%$ PK/PD target attainment against Enterobacterales and P. aeruginosa strains with an MIC value of up to $4 \mu \mathrm{g} / \mathrm{mL}$ [40, 42].

In an in vitro hollow-fibre infection model, drug exposures corresponding to imipenem $500 \mathrm{mg}$ with relebactam $250 \mathrm{mg}$ every $6 \mathrm{~h}$ as a $30 \mathrm{~min}$ infusion produced sustained bactericidal activity against KPC-producing $K$. pneumoniae and imipenem-resistant $P$. aeruginosa strains $[33,39]$. This exposure dose was effective against ten Enterobacterales strains producing various class $\mathrm{A}$ and class $\mathrm{C} \beta$-lactamases $[33,39]$. Moreover, at simulated human exposures of imipenem $500 \mathrm{mg}$ plus relebactam $250 \mathrm{mg}$ every $6 \mathrm{~h}$ for 7 days, bacterial killing against Enterobacterales, both KPC-producing strains and AmpC-producing strains with porin loss, was $>4 \log _{10}$ kill over the first $24 \mathrm{~h}$ and there was no regrowth [43]. Against $P$. aeruginosa isolates, including phenotypes that hyperexpressed AmpC or exhibited OprD mutation with porin loss, there was an initial 3-4 $\log _{10}$ kill over the first $24 \mathrm{~h}$, followed by subsequent modest regrowth of $P$. aeruginosa observed over 14 days of imipenem plus relebactam exposure [43]. 


\subsection{Resistance}

The most common mechanism of resistance to imipenem/relebactam is the production of $\beta$-lactamases that are not inhibited by relebactam, such as MBLs or oxacillinases [2, 9, 24, 44]. For example, in the SMART 2015-2017 surveillance program, 271 of 17,911 Enterobacterales isolates and 120 of 1959 P. aeruginosa isolates collected in Europe [24] and 34 of 6671 Enterobacterales isolates and 29 of 846 P. aeruginosa isolates collected in the USA [45] were nonsusceptible to imipenem/relebactam. In Europe, 96\% of 271 imipenem/ relebactam-nonsusceptible Enterobacterales isolates carried MBL-type and/or OXA-48-like carbapenemases, while $72 \%$ of 120 imipenem/relebactam-nonsusceptible $P$. aeruginosa isolates carried MBLs and 15\% carried class A Guiana extended-spectrum (GES) $\beta$-lactamases [24]. In the USA, $18 \%$ of 34 imipenem/relebactam-nonsusceptible Enterobacterales carried MBL-type and/or OXA-48-like carbapenemases and $14 \%$ of 29 P. aeruginosa isolates carried MBLs, including one isolate that co-carried GES $\beta$-lactamases [45]. In addition to the expression of $\beta$-lactamases that are not inhibited by relebactam, other potential resistance mechanisms to imipenem/relebactam include altered permeability or (over)expression of efflux pumps [44].

Although the risk for resistance development cannot be excluded, spontaneous resistance against imipenem/relebactam appeared to occur at a very low rate against $P$ seudomonas and most KPC-expressing Klebsiella species [33].

\subsection{Other Effects}

In healthy subjects $(n=36)$, a single supratherapeutic dose (4.6-fold higher) of intravenous relebactam did not prolong the corrected QT interval [46]. In preclinical studies, there were no cardiovascular, respiratory or gastrointestinal effects of concern reported for imipenem/cilastatin [33].

\section{Pharmacokinetic Properties}

The pharmacokinetics of imipenem/cilastatin are not affected when coadministered with relebactam [47] and their pharmacokinetic properties are also complementary to each other [40]. The pharmacokinetics of imipenem/cilastatin/relebactam are best described by a two-compartment model of disposition with zero-order intravenous infusion and linear first-order elimination [40].

The peak plasma concentration $\left(\mathrm{C}_{\max }\right)$ and AUC of imipenem, cilastatin and relebactam increase in a dose-proportional manner $[11,12]$. In healthy males with normal renal function, minimal accumulation was observed following multiple intravenous infusions of imipenem/cilastatin plus relebactam [12]. Following multiple 30-min intravenous infusions of imipenem/cilastatin 500/500 mg plus relebactam $250 \mathrm{mg}$ every $6 \mathrm{~h}$ in patients with active bacterial infections, steady-state $\mathrm{C}_{\max }$ of imipenem and relebactam were $88.9 \mu \mathrm{M}$ and $58.5 \mu \mathrm{M}$, respectively, with the respective AUC from time zero to $24 \mathrm{~h}$ being $500 \mu \mathrm{M} \cdot \mathrm{h}$ and $390.5 \mu \mathrm{M} \cdot \mathrm{h}[11$, 12].

Plasma protein binding is $\approx 20 \%, 40 \%$ and $22 \%$ for imipenem, cilastatin and relebactam, respectively; the plasma protein binding of relebactam is independent of drug concentration $[11,12]$. At steady-state, volume of distribution of imipenem, cilastatin, and relebactam is $24.3 \mathrm{~L}, 13.8 \mathrm{~L}$ and $19.0 \mathrm{~L}$, respectively $[11,12]$. A phase I study in otherwise healthy volunteers showed that both imipenem and relebactam have good intrapulmonary penetration when administered in combination, with relative exposures in bronchial epithelial lining fluid versus plasma being $55 \%$ and $54 \%$, respectively [48].

Imipenem is extensively metabolized in the kidneys by dehydropeptidase-I, and to prevent it from being metabolized too quickly, imipenem is coadministered with cilastatin, a dehydropeptidase-I inhibitor, which limits the renal metabolism of imipenem [11, 12]. Relebactam is minimally metabolized [11, 12].

Imipenem, cilastatin and relebactam are mainly excreted renally, involving both glomerular filtration and active tubular secretion [11, 12]. Following multipledose administrations in healthy volunteers, $\approx 63 \%, 77 \%$ and $>90 \%$ of the administered imipenem, cilastatin and relebactam doses were recovered as unchanged drug in human urine. The mean terminal elimination half-lives of imipenem/cilastatin and relebactam are $1.0 \mathrm{~h}$ and $1.2 \mathrm{~h}$, respectively [11, 12].

Sex, race, age and weight have no clinically relevant effects on the pharmacokinetics of imipenem, cilastatin and relebactam $[11,12,40]$. Hepatic impairment is not expected to have any clinically relevant impact on imipenem/cilastatin/relebactam exposure, as the drugs are primarily excreted renally. Relative to healthy people with normal renal function, imipenem and relebactam exposure in patients with mild, moderate or severe renal impairment was 1.22- to 2.01-fold and 1.38- to 3.05-fold higher, respectively [40]; dosage adjustment of imipenem/cilastatin/relebactam is therefore required in patients with renal impairment [11, 12].

Given that imipenem, cilastatin and relebactam are mostly recovered as unchanged drug in urine, drug-drug interactions with imipenem/cilastatin/relebactam driven by CYP inhibition or induction are unlikely $[11,12]$. Although relebactam is a substrate of OAT3, OAT4, MATE1 and MATE2K transporters [11, 12], there were no clinically significant differences in the pharmacokinetics of imipenem/cilastatin/relebactam when it was coadministered with probenecid, an inhibitor of OAT3 [49]. 
Based on reports of the concomitant use of imipenem/ cilastatin with the anticonvulsant valproic acid/divalprox sodium or the antiviral ganciclovir, coadministration of imipenem/cilastatin/relebactam with these drugs is not recommended [11, 12]. Coadministration of valproic acid or divalprox sodium with carbapenems, such as imipenem, is not recommended as it may reduce concentrations of valproic acid, thereby increasing the risk of breakthrough seizures in patients with seizure disorders; supplemental anticonvulsant therapy should be considered if coadministration with imipenem/cilastatin/relebactam is necessary. In addition, generalized seizures have been reported when ganciclovir was coadministered with imipenem/cilastatin; concomitant use should be avoided unless the potential benefits outweigh the risks $[11,12]$.

Imipenem/cilastatin/relebactam is compatible to be coadministered with other antibacterial agents $[11,12,50]$ or with several antifungals, such as anidulafungin, micafungin, caspofungin and fluconazole [50].

\section{Therapeutic Efficacy}

The efficacy of imipenem/cilastatin/relebactam was initially established in two dose-ranging phase II trials for the treatment of adults (aged $\geq 18$ years) with cUTI (MK7655-003 [51]; Sect. 4.1) or cIAI (MK7655-004 [52]; Sect. 4.2). The efficacy of imipenem/cilastatin/relebactam in the treatment of adults (aged $\geq 18$ years) with HABP/ VABP was evaluated in the phase III RESTORE IMI-2 trial (Sect. 4.3 53]), while the efficacy of this drug combination for the treatment of imipenem-nonsusceptible infections, including cUTI, cIAI and HABP/VABP, was evaluated in the phase III RESTORE IMI-1 trial (Sect. 4.4 [54]). All of these trials were randomized, double-blinded, multinational controlled trials [51-54].

In the phase II trials, relebactam doses of $125 \mathrm{mg}$ and 250 $\mathrm{mg}$ were evaluated [51, 52], whereas in the phase III trials, a relebactam dose of $250 \mathrm{mg}$ was selected on the basis of simulations using data from MK7655-004 (Sect. 4.2) and three phase I studies [53, 54]. All doses of relebactam were coadministered with imipenem/cilastatin 500/500 mg [51-54].

\subsection{Complicated Urinary Tract Infections}

In MK7655-003, patients with cUTIs (including acute pyelonephritis) requiring hospitalization and intravenous antibacterial therapy were randomized to receive relebactam $125 \mathrm{mg}$ [ $n=79$ microbiologically evaluable (ME) patients], $250 \mathrm{mg}(n=71)$ or placebo $(n=80)$, each coadministered with imipenem/cilastatin 500/500 mg every $6 \mathrm{~h}$ as a 30-min intravenous infusion for 4-14 days [51].
Both doses of relebactam plus imipenem/cilastatin were noninferior (based on prespecified criteria) to imipenem/ cilastatin plus placebo in terms of the proportion of ME patients with a favourable microbiological response at the discontinuation of intravenous therapy (DCIV) visit (98.6\% and $95.5 \%$ vs $98.7 \%$; primary endpoint). In addition, all $23 \mathrm{ME}$ patients infected with imipenem-nonsusceptible pathogens across the three treatment groups had a favourable microbiological response at the DCIV visit. Favourable microbiological response was pathogen eradication, defined as a urine culture taken at DCIV showing all baseline uropathogens with $\geq 10^{5}$ colony forming units $(\mathrm{CFU}) / \mathrm{mL}$ found reduced to $<10^{4} \mathrm{CFU} / \mathrm{mL}$ [51].

\subsection{Complicated Intra-Abdominal Infections}

In MK7655-004, patients with cIAIs were randomized to receive relebactam $125 \mathrm{mg}$ ( $n=87 \mathrm{ME}$ patients), $250 \mathrm{mg}$ $(n=83)$ or placebo $(n=85)$, each coadministered with imipenem/cilastatin 500/500 $\mathrm{mg}$ every $6 \mathrm{~h}$ as a $30-\mathrm{min}$ intravenous infusion for 4-14 days [52].

Both doses of relebactam plus imipenem/cilastatin were noninferior (both $p<0.001$ ) to imipenem/cilastatin plus placebo in terms of the proportion of ME patients with a favourable clinical response at the DCIV visit $(98.8 \%$ and $96.3 \%$ vs $95.2 \%$; primary endpoint) [52]. In addition, all $34 \mathrm{ME}$ patients infected with imipenem-nonsusceptible pathogens across the three treatment groups had a favourable clinical response at the DCIV visit. Favourable clinical response was defined as resolution of all or most presenting signs and symptoms of IAIs with no need for further antibiotic therapy [52].

\subsection{Hospital-Acquired or Ventilator-Associated Bacterial Pneumonia}

RESTORE IMI-2 evaluated the noninferiority of imipenem/ cilastatin/relebactam to piperacillin/tazobactam for the treatment of HABP/VABP in adults [53]. Hospitalized patients requiring antibiotic therapy for the treatment of nonventilated HABP, ventilated HABP or VABP were randomized to receive imipenem/cilastatin/relebactam 500/500/250 mg $(n=268)$ or piperacillin/tazobactam $4 \mathrm{~g} / 500 \mathrm{mg}(n=269)$ every $6 \mathrm{~h}$ as a 30-min intravenous infusion for 7-14 days; doses were adjusted based on renal function. In addition, empiric intravenous linezolid was added to both treatment regimens until baseline respiratory cultures confirmed the absence of methicillin-resistant Staphylococcus aureus. Randomization was stratified based on diagnosis (nonventilated HABP vs ventilated HABP/VABP) and Acute Physiology and Chronic Health Evaluation II (APACHE II) score $(<15$ vs $\geq 15$ ) [53]. 
The primary endpoint and key secondary endpoint were day 28 all-cause mortality rate and favourable clinical response at the early follow-up visit (EFU; 7-14 days after the end of therapy) in the modified intent-to-treat (MITT) population, respectively; noninferiority of imipenem/cilastatin/relebactam to piperacillin/tazobactam was tested in both the primary and key secondary endpoint analyses [53].

Baseline characteristics were well balanced between the treatment groups [53]. More than half of MITT patients $(66.1 \%)$ were in the intensive care unit, $47.5 \%$ had APACHE II score $\geq 15,48.6 \%$ had ventilated HABP/VABP and $42.9 \%$ were aged $>65$ years. The most common baseline lower respiratory tract pathogen included K. pneumoniae $(25.6 \%)$, P. aeruginosa (18.9\%), A. calcoaceticus-baumannii complex (15.7\%) and E. coli (15.5\%). Among patients with relevant data available, $79.7 \%$ of 187 patients in the imipenem/ cilastatin/relebactam group and $65.8 \%$ of 193 patients in the piperacillin/tazobactam group had all gram-negative baseline pathogens (including multiple pathogens in the case of polymicrobial infections) susceptible to the randomized study treatment [53].

Imipenem/cilastatin/relebactam was noninferior to piperacillin/tazobactam for the primary endpoint of day 28 allcause mortality rate (adjusted treatment difference $-5.3 \%$; $95 \% \mathrm{CI}-11.9$ to $1.2 \%$ ) and the key secondary endpoint of favourable clinical response at EFU $(5.0 \% ;-3.2$ to $13.2 \%)$ in the MITT population (Fig. 1) [53].

In predefined subgroup analyses, based on $95 \%$ confidence intervals (CIs), the day 28 mortality rate was lower with imipenem/cilastatin/relebactam than with piperacillin/ tazobactam in subgroups of patients with mechanically ventilated HABP/VABP or those with an APACHE II score of $\geq 15$, both particularly high-risk critically ill populations [53]. Similarly, the favourable clinical response rate at EFU was higher with imipenem/cilastatin/relebactam than with piperacillin/tazobactam in the subgroup of patients with an APACHE II score of $\geq 15$. In the other evaluated clinically relevant subgroups including age ( $<65$ or $\geq 65$ years), sex (male or female), renal impairment, concurrent bacteraemia, clinical pulmonary infection score, geographic region, prior systemic gram-negative therapy, concomitant systemic gram-negative therapy and key baseline pathogens, day 28 mortality rate and favourable clinical response rate at EFU were comparable between the treatment groups [53]. Of note, in a post hoc multivariate analysis of RESTORE IMI2 , age $\geq 65$ years, mechanical ventilation, APACHE II score of $\geq 15$ and renal impairment were identified as independent negative predictors of all-cause mortality rates and clinical response rates at EFU, regardless of treatment group [55], and demonstrated no interactions between these predictors and treatment assignment.

In microbiological MITT (mMITT) patients who had $\geq 1$ baseline lower respiratory tract pathogen with

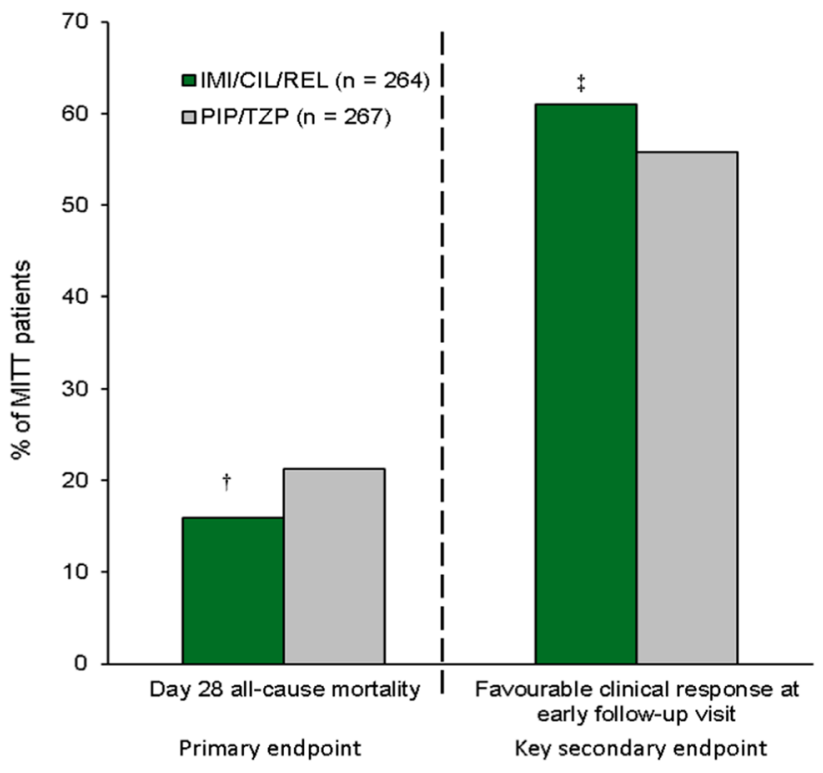

Fig. 1 Efficacy of imipenem/cilastatin/relebactam in RESTORE-IMI 2 in adults with hospital-acquired or ventilator-associated bacterial pneumonia [53]. 'Noninferior to PIP/TAZ as the upper limit of the 2-sided 95\% CI for the adjusted treatment difference did not exceed $10 \%,{ }^{\ddagger}$ Noninferior to PIP/TAZ as the lower limit of the 2-sided $95 \%$ CI for the adjusted treatment difference was greater than $-12.5 \%$. IMI/CIL/REL imipenem/cilastatin/relebactam, MITT modified intentto-treat, $P I P / T Z P$ piperacillin/tazobactam.

laboratory-confirmed in vitro susceptibility to both imipenem/relebactam and piperacillin/tazobactam, per-pathogen efficacy outcomes were generally comparable between the treatment groups [56]. For example, in imipenem/cilastatin/ relebactam and piperacillin/tazobactam recipients, day 28 all-cause mortality rate and favourable clinical response rate at EFU were $16.7 \%$ and $62.1 \%$ versus $22.5 \%$ and $58.9 \%$ for Enterobacterales ( $n=132$ and 129), 32.4\% and $41.2 \%$ versus $14.6 \%$ and $60.4 \%$ for $P$. aeruginosa $(n=34$ and 48$)$ and $15.6 \%$ and $59.4 \%$ versus $22.2 \%$ and $58.3 \%$ for A. calcoaceticus-baumannii complex ( $n=32$ and 36). The numerical difference in mortality rates and clinical response against $P$. aeruginosa could be due to small sample size and uneven distribution of patients infected with this pathogen between the treatment groups [56]. It should also be noted that $P$. aeruginosa was an independent predictor of lower clinical response rates, regardless of treatment group [55].

\subsection{Infections Specifically Caused by Imipenem-Nonsusceptible Pathogens}

RESTORE IMI-1 was a descriptive study that specifically focused on the efficacy of imipenem/cilastatin/relebactam in the treatment of infections caused by carbapenem-nonsusceptible pathogens [54]. Eligible patients were hospitalized 
adults with HABP/VABP, cUTIs or cIAIs caused by imipenem-nonsusceptible but colistin- and imipenem/relebactamsusceptible pathogens who had previously failed antibacterial therapy; pathogen susceptibility was determined by local prescreening susceptibility testing and subsequently confirmed at a central laboratory. Key exclusion criteria included APACHE II score $>30$, creatinine clearance $\left(\mathrm{CL}_{\mathrm{CR}}\right)<15 \mathrm{~mL} / \mathrm{min}$ or requiring haemodialysis/peritoneal dialysis, pulmonary obstructions in patients with HABP/ VABP and complete obstruction of any portion of the urinary tract in patients with cUTIs. Patients receiving concomitant systemic or inhaled agents against Enterobacterales, Pseudomonas species and gram-negative anaerobic bacilli were also among those excluded [54].

Enrolled patients $(n=47)$ were stratified by infection type and were randomized to receive intravenous imipenem/cilastatin/relebactam 500/500/250 mg every $6 \mathrm{~h}$ plus placebo or intravenous imipenem/cilastatin $500 / 500 \mathrm{mg}$ every $6 \mathrm{~h}$ plus intravenous colistin (loading dose to achieve $300 \mathrm{mg}$ colistin base activity, followed by up to $150 \mathrm{mg}$ colistin base activity as maintenance doses) every $12 \mathrm{~h}$ for 5-21 days for cUTI and cIAI or 7-21 days for HABP/ VABP [54]. Imipenem/cilastatin/relebactam plus placebo and imipenem/cilastatin plus colistin were administered as a 30-min intravenous infusion and dosages were adjusted based on renal function. The primary endpoint was the favourable overall response in the mMITT population (i.e. all randomized patients who received $\geq 1$ dose of study drug and with $\geq 1$ qualifying pathogen as confirmed by central laboratory test results). Favourable overall response was defined as survival through day 28 for HABP/VABP, clinical response at day 28 for cIAI and composite clinical and microbiological response 5-9 days after the end of therapy for cUTI [54].

Baseline patient, disease and microbiological characteristics were generally similar between treatment groups [54]. In the mMITT population $(n=31), 11$ patients had HABP/ VABP, 16 had cUTI and 4 had cIAI. At baseline, 35\% of patients were aged $\geq 65$ years, $29 \%$ had APACHE II scores $>15$ and $23 \%$ had $\mathrm{CL}_{\mathrm{CR}}<60 \mathrm{~mL} / \mathrm{min}$. The most common qualifying baseline pathogen was $P$. aeruginosa (77\%), followed by Klebsiella species (16\%) and other Enterobacterales $(6 \%)$, and detected $\beta$-lactamases were mostly AmpC (84\%), followed by ESBLs (35\%), KPC (16\%) and OXA-48 (3\%). Mean treatment duration in the imipenem/cilastatin/ relebactam and imipenem/cilastatin plus colistin groups was 11.4 and 10.8 days, respectively [54].

Overall, treatment with imipenem/cilastatin/relebactam or imipenem/cilastatin plus colistin was effective for treating imipenem-nonsusceptible gram-negative infections, with $71.4 \%$ and $70.2 \%$ of mMITT patients in the respective treatment groups achieving a favourable overall response (primary endpoint) [54]. In the respective treatment groups, most mMITT patients with HABP/VABP $(87.5 \%$ vs $66.7 \%)$ or cUTI $(72.7 \%$ vs $100 \%)$, but none of the four participants with cIAI achieved a favourable overall response. Per-pathogen favourable overall response rates in imipenem/cilastatin/ relebactam versus imipenem/cilastatin plus colistin recipients were $40 \%$ versus $100 \%$ for Enterobacterales $(n=5$ and 2 ) and $81 \%$ versus $63 \%$ for $P$. aeruginosa $(n=16$ and 8$)$. In addition, although the study was not powered to detect statistically significant differences, patients treated with imipenem/cilastatin/relebactam had numerically higher favourable clinical response rates (i.e. resolution of baseline signs and symptoms) at day 28 and lower day 28 mortality rates than those treated with imipenem/cilastatin plus colistin (adjusted treatment difference: $26.3 \%$ and $-17.3 \%$, respectively) [54].

The efficacy outcomes were consistent when treatment response was evaluated in patients with qualifying baseline pathogens identified based only on local microbiology laboratory culture and susceptibility results (i.e. the supplemental mMITT population; $n=41$ ) [57].

\section{Tolerability}

Based on extensive evidence from its use in clinical practice, imipenem/cilastatin is considered to have a well-established safety profile [33]. In the clinical trials discussed in Sect. 4, imipenem/cilastatin/relebactam was generally well tolerated in patients with cUTI, cIAI or HABP/VABP, with the safety profile consistent with that established for imipenem/cilastatin [33, 51-54]. The nature and frequency of adverse events occurring with imipenem/cilastatin/relebactam were generally similar in patients with HABP/VABP, cUTIs or cIAIs [51-53]. Discussion in this section focuses largely on data available from RESTORE IMI-2 (Sect. 4.3) [53].

In RESTORE IMI-2, treatment-related adverse events (TRAEs) occurred in $11.7 \%$ of 266 imipenem/cilastatin/ relebactam recipients and $9.7 \%$ of 269 piperacillin/tazobactam recipients [53]. The most common TRAEs ( $>2 \%$ incidence) in the respective groups were diarrhoea (2.3\% vs $2.2 \%)$, increased alanine aminotransferase (2.3\% vs $1.1 \%)$ and increased aspartate aminotransferase $(2.3 \%$ vs $0 \%)$. Serious TRAEs were reported in $1.1 \%$ of imipenem/cilastatin/ relebactam and $0.7 \%$ of piperacillin/tazobactam recipients, while $2.3 \%$ and $1.5 \%$ of patients in the respective group discontinued treatment due to TRAEs. No deaths were considered treatment-related [53].

In RESTORE IMI-2, treatment-related renal impairment was rare in imipenem/cilastatin/relebactam and piperacillin/ tazobactam recipients ( $0 \%$ and $0.4 \%$, respectively) [53]. Furthermore, in RESTORE IMI-1, imipenem/cilastatin/relebactam was associated with a more favourable renal safety profile compared with colistin-based therapy, as demonstrated by a significantly lower incidence of treatment-emergent 
nephrotoxicity observed with imipenem/cilastatin/relebactam than with colistin-based therapy $(10 \%$ vs $56 \% ; p=$ $0.002)$ in a prospectively specified analysis $[54,58]$.

As seen with nearly all antibacterial agents, cases of Clostridioides difficile-associated diarrhoea (CDAD) have been reported with imipenem/cilastatin/relebactam [11, 12]. The severity of CDAD may range from mild diarrhoea to fatal colitis. In all patients presenting with diarrhoea during or following treatment with imipenem/cilastatin/relebactam, CDAD must be considered, as it has been reported to occur over 2 months after the administration of antibacterial agents. If CDAD is suspected or confirmed, treatment discontinuation of imipenem/cilastatin/relebactam and appropriate clinical management (e.g. maintaining proper fluid and electrolyte levels, protein supplementation and antibacterial treatment for $C$. difficile [11]) should be considered $[11,12]$.

Although CNS adverse reactions, such as seizures, confusional states and mycolonic activity, did not occur during the clinical trials with imipenem/cilastatin/relebactam, there have been reports of such CNS adverse reactions occurring in patients who received imipenem/cilastatin, a component of imipenem/cilastatin/relebactam, especially when the recommended dosages were exceeded [11, 12]. These CNS adverse reactions have been reported most commonly in patients with preexisting CNS disorders (e.g. brain lesions or history of seizures) and/or those with compromised renal function $[11,12]$. In the case of CNS adverse reactions, patients should undergo a neurological evaluation to determine whether imipenem/cilastatin/relebactam should be discontinued [11].

\section{Dosage and Administration}

In the USA, imipenem/cilastatin/relebactam is approved for the treatment of adults with HABP/VABP, and also for the treatment of cUTIs (including pyelonephritis) and cIAIs caused by susceptible gram-negative organisms in adults with limited or no alternative treatment options [11]. In the $\mathrm{EU}$, the combination is approved the treatment of adults with HABP/VABP or bacteraemia that occurs in association with, or is suspected to be associated with, HABP/VABP, and infections caused by aerobic gram-negative organisms in adults with limited treatment options [12].

The approved dosage of imipenem/cilastatin/relebactam is $1.25 \mathrm{~g}$ (imipenem $500 \mathrm{mg} /$ cilastatin $500 \mathrm{mg} /$ relebactam $250 \mathrm{mg}$ ) once every $6 \mathrm{~h}$, administered by an intravenous infusion over $30 \mathrm{~min}[11,12]$. The total recommended treatment duration is 4-14 days in the USA [11] and 5-14 days in the EU [12], based on the type and severity of infection, as well as clinical response to treatment. The dosage of imipenem/cilastatin/relebactam should be reduced in patients with estimated $\mathrm{CL}_{\mathrm{CR}}<90 \mathrm{~mL} / \mathrm{min}$, with the recommended dosage dependent on the degree of renal impairment; $\mathrm{CL}_{\mathrm{CR}}$ should be monitored in patients with fluctuating renal function. In patients with end-stage renal disease on haemodialysis, imipenem/cilastatin/relebactam should be administered after a haemodialysis session [11, 12].

Local prescribing information should be consulted for detailed information regarding the use of imipenem/cilastatin/relebactam, including contraindications, precautions and warnings, use in special populations and potential drug interactions.

\section{Place of Imipenem/Cilastatin/Relebactam in the Management of Gram-Negative Infections}

The clinical and economic burden of treating patients with carbapenem-resistant gram-negative infections is substantial $[3,5,59,60]$. Subsequently, optimizing antibacterial therapy through antimicrobial stewardship is important to maximize clinical outcomes while reducing the economic burden and minimizing the unintended consequences of antibacterial use (e.g. toxicity, resistance development) [6, 7, 61-65]. The choice of appropriate empirical antibacterial therapy is based on several factors, including the type of infection, causative pathogen, local antibacterial resistance patterns, patient characteristics (e.g. presence of comorbidities, prior history of antibiotic therapy) and drug characteristics (e.g. potential drug-drug interactions, efficacy and safety profiles and cost) $[6,7,61-65]$.

Current guidelines [61-65] and treatment guidances [6, 7] recommend fluoroquinolones, aminoglycosides, cefiderocol, tigecycline and $\beta$-lactam/ $\beta$-lactamase inhibitor combinations (e.g. ceftolozane/tazobactam, meropenem/ vaborbactam, ceftazidime/avibactam or imipenem/cilastatin/relebactam) as preferred or alternative treatment options, depending on the type of infection and causative pathogen(s), for the management of gram-negative bacterial infections. Although the approval of imipenem/ cilastatin/relebactam is too recent to have been included in most major guidelines for the treatment of HABP/VABP or cIAI [61-64], imipenem/cilastatin/relebactam is recommended as an alternative treatment option for cUTI, especially for infection caused by MDR pathogens, in the 2020 European Association of Urology guidelines on urological infections [65]. Furthermore, the 2020 antimicrobial resistance treatment guidance from Infectious Diseases Society of America (IDSA) recommends imipenem/cilastatin/relebactam, along with ceftazidime/ avibactam and meropenem/vaborbactam, as a preferred treatment option for infections (excluding cystitis) caused 
by carbapenem-resistant Enterobacterales; however, its use is not recommended for carbapenem-resistant Enterobacterales that produce MBLs or OXA-48-like carbapenemases. IDSA antimicrobial resistance treatment guidance also recommends imipenem/cilastatin/relebactam as one of the preferred treatment options for the management of $P$. aeruginosa infections with difficult-to-treat resistance (defined as nonsusceptible to piperacillin/tazobactam, ceftazidime, cefepime, aztreonam, meropenem, imipenem/ cilastatin, ciprofloxacin, and levofloxacin) [6]

Imipenem/cilastatin/relebactam has been approved in the USA and EU for the treatment of infections caused by gram-negative organisms in adults, including those with limited or no alternative treatment options (Sect. 6). When combined with the novel $\beta$-lactamase inhibitor relebactam, the antibacterial activity of imipenem against gram-negative pathogens expressing class $\mathrm{A}$ and/or $\mathrm{C} \beta$-lactamases is restored (Sect. 2). Imipenem/relebactam exhibited broad, potent in vitro activity against Enterobacterales and $P$. aeruginosa, including many $\beta$-lactam-nonsusceptible, MDR or KPC-positive strains (Sect. 2.2).

In the clinical setting, the antibacterial efficacy of imipenem/ cilastatin/relebactam for the treatment of gram-negative infections in adults was initially demonstrated in phase II studies, where imipenem/cilastatin plus relebactam was noninferior to imipenem/cilastatin in the treatment of cUTI (Sect. 4.1) and cIAI (Sect. 4.2). Furthermore, in the phase III RESTOREIMI 2 study, imipenem/cilastatin/relebactam was noninferior to piperacillin/tazobactam for the treatment of HABP/VABP, with the day 28 mortality rate being lower with imipenem/ cilastatin/relebactam than with piperacillin/tazobactam in critically ill and/or high-risk populations (Sect.4.3). In addition, although not powered to assess statistical significance, results from the phase III RESTORE IMI-1 study in adults with infections caused by imipenem-nonsusceptible pathogens indicated that imipenem/cilastatin/relebactam treatment was associated with a numerically higher clinical cure rate than colistin-based therapy (Sect. 4.4).

Imipenem/cilastatin/relebactam was generally well tolerated in patients with cUTI, cIAI or HABP/VABP in clinical trials, with a safety profile consistent with that established for imipenem/cilastatin (Sect. 5). Moreover, imipenem/cilastatin/relebactam was associated with a more favourable renal safety profile compared with colistin-based therapy $[54,58]$.

In conclusion, although ongoing clinical experience with imipenem/cilastatin/relebactam will further determine its role with respect to other available antibacterial therapies, current evidence indicates that imipenem/cilastatin/relebactam is an effective and generally well tolerated option for gram-negative infections in adults, including critically ill and/or high-risk patients, and a potential therapy for infections caused by carbapenem-resistant pathogens.
Data Selection Imipenem/cilastatin/relebactam: 312 records identified

Duplicates removed

Excluded during initial screening (e.g. press releases; news reports; not relevant drug/indication; preclinical study; reviews; case reports; not randomized trial)

Excluded during writing (e.g. reviews; duplicate data; small patient number; nonrandomized/phase I/II trials)

Cited efficacy/tolerability articles

Cited articles not efficacy/tolerability

Search Strategy: EMBASE, MEDLINE and PubMed from 1946 to present. Clinical trial registries/databases and websites were also searched for relevant data. Key words were imipenem, cilastin, relebactam, Recarbrio, gram-negative bacterial infection. Records were limited to those in English language. Searches last updated 3 Feb 2021

Acknowledgements During the peer review process, the manufacturer of imipenem/cilastatin/relebactam was also offered an opportunity to review this article. Changes resulting from comments received were made on the basis of scientific and editorial merit.

\section{Declarations}

Funding The preparation of this review was not supported by any external funding.

Authorship and Conflict of interest Young-A Heo is a salaried employee of Adis International Ltd/Springer Nature and declares no relevant conflicts of interest. Young-A Heo contributed to the review and is responsible for the article content.

Ethics approval, Consent to participate, Consent to publish, Availability of data and material, Code availability Not applicable.

\section{References}

1. World Health Organization. Global priority list of antibioticresistant bacteria to guide research, discovery, and development of new antibiotics. 2017. https://www.who.int. Accessed $21 \mathrm{Dec}$ 2020.

2. Zhanel GG, Lawrence $\mathrm{CK}$, Adam $\mathrm{H}$, et al. Imipenem-relebactam and meropenem-vaborbactam: two novel carbapenem-beta-lactamase inhibitor combinations. Drugs. 2018;78(1):65-98.

3. Tabak YP, Sung A, Ye G, et al. Attributable burden in patients with carbapenem-nonsusceptible gram-negative respiratory infections. PLoS ONE. 2020;15(2):e0229393.

4. McCann E, Srinivasan A, DeRyke C, et al. Carbapenem-nonsusceptible gram-negative pathogens in ICU and non-ICU settings in US hospitals in 2017: a multicenter study. Open Forum Infect Dis. 2018;5(10):241.

5. Tabak YP, Sung AH, Ye G, et al. Attributable clinical and economic burden of carbapenem-non-susceptible gram-negative 
infections in patients hospitalized with complicated urinary tract infections. J Hosp Infect. 2019;102(1):37-44.

6. Tamma PD, Aitken SL, Bonomo RA, et al. Infectious Diseases Society of America antimicrobial resistant treatment guidance: gram-negative bacterial infections. 2020. https://idsociety.org. Accessed 21 Dec 2020.

7. Hawkey PM, Warren RE, Livermore DM, et al. Treatment of infections caused by multidrug-resistant gram-negative bacteria: report of the British Society for Antimicrobial Chemotherapy/ Healthcare Infection Society/British Infection Association joint Working Party. J Antimicrob Chemother. 2018;73(Suppl 3):iii2-78.

8. Asempa TE, Nicolau DP, Kuti JL. Carbapenem-nonsusceptible Pseudomonas aeruginosa isolates from intensive care units in the United States: a potential role for new beta-lactam combination agents. J Clin Microbiol. 2019;57(8):1-9.

9. Wong D, van Duin D. Novel beta-lactamase inhibitors: unlocking their potential in therapy. Drugs. 2017;77(6):615-28.

10. Schmidt-Malan SM, Mishra AJ, Mushtaq A, et al. In vitro activity of imipenem-relebactam and ceftolozane-tazobactam against resistant gram-negative bacilli. Antimicrob Agents Chemother. 2018;62(8):1-10.

11. Merck Sharp \& Dohme. Recarbrio ${ }^{\mathrm{TM}}$ (imipenem, cilastatin, and relebactam): US prescribing information. 2020. https://www. fda.gov. Accessed 23 Dec 2020.

12. Merck Sharp \& Dohme. Recarbrio: EU summary of product characteristics. 2021. https://www.ema.europa.eu. Accessed 3 Feb 2021.

13. Papp-Wallace KM, Barnes MD, Alsop J, et al. Relebactam is a potent inhibitor of the KPC-2 beta-lactamase and restores imipenem susceptibility in KPC-producing Enterobacteriaceae. Antimicrob Agents Chemother. 2018;62(6):1-9.

14. Barnes MD, Bethel CR, Alsop J, et al. Inactivation of the Pseudomonas-derived cephalosporinase-3 (PDC-3) by relebactam. Antimicrob Agents Chemother. 2018;62(5):1-6.

15. Gomez-Simmonds A, Stump S, Giddins MJ, et al. Clonal background, resistance gene profile, and porin gene mutations modulate in vitro susceptibility to imipenem-relebactam in diverse Enterobacteriaceae. Antimicrob Agents Chemother. 2018;62(8):1-10.

16. Hirsch EB, Ledesma KR, Chang KT, et al. In vitro activity of MK-7655, a novel beta-lactamase inhibitor, in combination with imipenem against carbapenem-resistant gram-negative bacteria. Antimicrob Agents Chemother. 2012;56(7):3753-7.

17. Lapuebla A, Abdallah M, Olafisoye O, et al. Activity of imipenem with relebactam against gram-negative pathogens from New York City. Antimicrob Agents Chemother. 2015;59(8):5029-31.

18. Carpenter J, Neidig N, Campbell A, et al. Activity of imipenem/relebactam against carbapenemase-producing Enterobacteriaceae with high colistin resistance. J Antimicrob Chemother. 2019;74(11):3260-3.

19. Chen IH, Nicolau DP, Kuti JL. Imipenem/cilastatin/relebactam alone and in combination against Pseudomonas aeruginosa in the in vitro pharmacodynamic model. Antimicrob Agents Chemother. 2020;64(12):e01764-e1820.

20. Young K, Painter RE, Raghoobar SL, et al. In vitro studies evaluating the activity of imipenem in combination with relebactam against Pseudomonas aeruginosa. BMC Microbiol. 2019;19(1):150.

21. Kazmierczak K, Depestel D, Young K, et al. In vitro activity of imipenem/relebactam against non-Morganellaceae Enterobacterales, Pseudomonas aeruginosa and Acinetobacter baumannii isolates from patients with respiratory tract infections in the United States: SMART 2016-2018 [abstract no. 3695 plus poster P4431]. In: 30th European Congress of Clinical Microbiology and Infectious Diseases. 2020.
22. Karlowsky JA, Lob SH, Kazmierczak KM, et al. In vitro activity of imipenem-relebactam against clinical isolates of gram-negative bacilli isolated in hospital laboratories in the United States as part of the SMART 2016 program. Antimicrob Agents Chemother. 2018;62(7):1-11.

23. Karlowsky JA, Lob SH, Raddatz J, et al. In vitro activity of imipenem/relebactam and ceftolozane/tazobactam against clinical isolates of gram-negative bacilli with difficult-to-treat resistance and multidrug-resistant phenotypes-SMART United States 2015-2017. Clin Infect Dis. 2020. https://doi.org/10.1093/cid/ ciaa381.

24. Lob SH, Karlowsky JA, Young K, et al. In vitro activity of imipenem-relebactam against resistant phenotypes of Enterobacteriaceae and Pseudomonas aeruginosa isolated from intraabdominal and urinary tract infection samples-SMART surveillance Europe 2015-2017. J Med Microbiol. 2020;69(2):207-17.

25. Yang Q, Zhang H, Yu Y, et al. In vitro activity of imipenem/relebactam against Enterobacteriaceae isolates obtained from intraabdominal, respiratory tract, and urinary tract infections in China: study for Monitoring Antimicrobial Resistance Trends (SMART), 2015-2018. Clin Infect Dis. 2020;71(Suppl 4):S427-35.

26. Lob S, Kazmierczak K, Hoban D, et al. Global activity of imipenem-relebactam and comparators against clinical gram-negative pathogens-SMART 2017 [abstract no. 1355]. Open Forum Infect Dis. 2018;5(Suppl 1):S415.

27. Karlowsky JA, Lob SH, Young K, et al. Activity of imipenem/ relebactam against Pseudomonas aeruginosa with antimicrobialresistant phenotypes from seven global regions: SMART 20152016. J Glob Antimicrob Resist. 2018;15:140-7.

28. Lob S, Kazmierczak K, Young K, et al. In vitro activity of imipenem/relebactam against gram-negative organisms collected globally from patients with different infection types: SMART 2018 [abstract no. 2375 plus poster P4428]. In: 30th European Congress of Clinical Microbiology and Infectious Diseases. 2020.

29. European Committee on Antimicrobial Susceptibility Testing. Breakpoint tables for interpretion of MICs and zone diameters version 10.0. . 2020. http://eucast.org/. Accessed 21 Dec 2020.

30. Clinical and Laboratory Standards Institute. Breakpoint Working Group report 2020. http://clsi.org/. Accessed 21 Dec 2020.

31. Canver MC, Satlin MJ, Westblade LF, et al. Activity of imipenemrelebactam and comparator agents against genetically characterized isolates of carbapenem-resistant Enterobacteriaceae. Antimicrob Agents Chemother. 2019;63(9):1-5.

32. Fraile-Ribot PA, Zamorano L, Orellana R, et al. Activity of imipenem-relebactam against a large collection of Pseudomonas aeruginosa clinical isolates and isogenic beta-lactam-resistant mutants. Antimicrob Agents Chemother. 2020;64(2):1-6.

33. European Medicines Agency. Recarbrio: EPAR-public assessment report 2020. https://www.ema.europa.eu. Accessed 21 Dec 2020.

34. Powles MA, Galgoci A, Misura A, et al. In vivo efficacy of relebactam (MK-7655) in combination with imipenem-cilastatin in murine infection models. Antimicrob Agents Chemother. 2018;62(8):e02577-e2617.

35. Mavridou E, Melchers RJ, van Mil AC, et al. Pharmacodynamics of imipenem in combination with beta-lactamase inhibitor MK7655 in a murine thigh model. Antimicrob Agents Chemother. 2015;59(2):790-5.

36. Patel M, Daryani N, Feng H, et al. Imipenem/relebactam pharmacokinetic/pharmacodynamic analyses from an in vivo neutropenic murine thigh infection model [abstract no. 1693 plus poster P3456]. In: 30th European Congress of Clinical Microbiology and Infectious Diseases. 2020.

37. Reyes S, Abdelraouf K, Nicolau DP. In vivo activity of humansimulated regimens of imipenem alone and in combination with relebactam against Pseudomonas aeruginosa in the murine thigh infection model. J Antimicrob Chemother. 2020;75(8):2197-205. 
38. Daryani N, Patel M, Flattery A, et al. Imipenem/relebactam pharmacokinetic/pharmacodynamic analyses from an in vivo neutropenic mouse delayed lung infection model [abstract no. 2086 plus poster 3457]. In: 30th European Congress of Clinical Microbiology and Infectious Diseases. 2020.

39. Wu J, Racine F, Wismer MK, et al. Exploring the pharmacokinetic/pharmacodynamic relationship of relebactam (MK-7655) in combination with imipenem in a hollow-fiber infection model. Antimicrob Agents Chemother. 2018;62(5):1-13.

40. Bhagunde P, Patel P, Lala M, et al. Population pharmacokinetic analysis for imipenem-relebactam in healthy volunteers and patients with bacterial infections. CPT Pharmacomet Syst Pharmacol. 2019;8(10):748-58.

41. Bhagunde $\mathrm{P}$, Zhang $\mathrm{Z}$, Racine $\mathrm{F}$, et al. A translational pharmacokinetic/pharmacodynamic model to characterize bacterial kill in the presence of imipenem-relebactam. Int J Infect Dis. 2019;89:55-61.

42. Patel M, Patel P, Daryani N, et al. Population pharmacokinetic/ pharmacodynamic assessment of a clinical imipenem/cilastatin and relebactam dose in patients with hospital-acquired bacterial pneumonia and ventilator-associated bacterial pneumonia [abstract no. 154 plus poster]. In: 30th European Congress of Clinical Microbiology and Infectious Diseases. 2020.

43. Noel AR, Bowker KE, Attwood M, et al. Antibacterial effect of imipenem/relebactam on aerobic gram-negative bacilli: in vitro simulations of 7 or 14 day human exposures. J Antimicrob Chemother. 2019;74(7):1945-51.

44. Johnston BD, Thuras P, Porter SB, et al. Activity of imipenemrelebactam against carbapenem-resistant Escherichia coli isolates from the United States in relation to clonal background, resistance genes, coresistance, and region. Antimicrob Agents Chemother. 2020;64(5):1-8.

45. Karlowsky JA, Lob SH, Kazmierczak KM, et al. In vitro activity of imipenem-relebactam against Enterobacteriaceae and Pseudomonas aeruginosa isolated from intraabdominal and urinary tract infection samples-SMART surveillance United States 2015-2017. J Glob Antimicrob Resist. 2020;21:223-8.

46. Boundy K, Liu Y, Bhagunde P, et al. Thorough QTc study of a single supratherapeutic dose of relebactam in healthy participants. Clin Pharmacol Drug Dev. 2020;9(4):466-75.

47. Rhee EG, Rizk ML, Calder N, et al. Pharmacokinetics, safety, and tolerability of single and multiple doses of relebactam, a beta-lactamase inhibitor, in combination with imipenem and cilastatin in healthy participants. Antimicrob Agents Chemother. 2018;62(9):e00280-e318.

48. Rizk ML, Rhee EG, Jumes PA, et al. Intrapulmonary pharmacokinetics of relebactam, a novel beta-lactamase inhibitor, dosed in combination with imipenem-cilastatin in healthy subjects. Antimicrob Agents Chemother. 2018;62(3):e01411-e1417.

49. Bhagunde P, Colon-Gonzalez F, Liu Y, et al. Impact of renal impairment and human organic anion transporter inhibition on pharmacokinetics, safety and tolerability of relebactam combined with imipenem and cilastatin. Br J Clin Pharmacol. 2020;86(5):944-57.

50. Ghazi IM, El Nekidy WS, Asay R, et al. Simultaneous administration of imipenem/cilastatin/relebactam with selected intravenous antimicrobials, a stewardship approach. PLoS ONE. 2020;15(5):e0233335

51. Sims M, Mariyanovski V, McLeroth P, et al. Prospective, randomized, double-blind, phase 2 dose-ranging study comparing efficacy and safety of imipenem/cilastatin plus relebactam with imipenem/cilastatin alone in patients with complicated urinary tract infections. J Antimicrob Chemother. 2017;72(9):2616-26.

52. Lucasti C, Vasile L, Sandesc D, et al. Phase 2, dose-ranging study of relebactam with imipenem-cilastatin in subjects with complicated intra-abdominal infection. Antimicrob Agents Chemother. 2016;60(10):6234-43.

53. Titov I, Wunderink RG, Roquilly A, et al. A randomized, doubleblind, multicenter trial comparing efficacy and safety of imipenem/cilastatin/relebactam versus piperacillin/tazobactam in adults with hospital-acquired or ventilator-associated bacterial pneumonia (RESTORE-IMI 2 study). Clin Infect Dis. 2020. https://doi. org/10.1093/cid/ciaa803.

54. Motsch J, de Oliveira CM, Stus V, et al. RESTORE-IMI 1: a multicenter, randomized, double-blind trial comparing efficacy and safety of imipenem/relebactam vs colistin plus imipenem in patients with imipenem-nonsusceptible bacterial infections. Clin Infect Dis. 2020;70(9):1799-808.

55. Tipping R, Du J, Losada MC, et al. Multivariate regression analysis to determine independent predictors of treatment outcomes in the RESTORE-IMI 2 trial [abstract]. In: IDWeek. 2020.

56. Losada MC, Maniar A, Du J, et al. Clinical and microbiologic outcomes by causative pathogen in hospital-acquired or ventilator-associated bacterial pneumonia (HABP/VABP) treated with imipenem/cilastatin(IMI)/relebactam (REL) versus piperacillin/ tazobactam (PIP/TAZ) [abstract]. In: IDWeek. 2020.

57. Kaye KS, Boucher HW, Brown ML, et al. Comparison of treatment outcomes between analysis populations in the RESTOREIMI 1 phase 3 trial of imipenem-cilastatin-relebactam versus colistin plus imipenem-cilastatin in patients with imipenem-nonsusceptible bacterial infections. Antimicrob Agents Chemother. 2020;64(5):1-10.

58. Brown ML, Motsch J, Kaye KS, et al. Evaluation of renal safety between imipenem/relebactam and colistin plus imipenem in patients with imipenem-nonsusceptible bacterial infections in the randomized, phase 3 RESTORE-IMI 1 study. Open Forum Infect Dis. 2020;7(3):1-7.

59. McCann E, Sung AH, Ye G, et al. Contributing factors to the clinical and economic burden of patients with laboratory-confirmed carbapenem-nonsusceptible gram-negative urinary tract infections. Clinicoecon Outcomes Res. 2020;12:191-200.

60. McCann E, Sung AH, Ye G, et al. Contributing factors to the clinical and economic burden of patients with laboratory-confirmed carbapenem-nonsusceptible gram-negative respiratory infections. Infect Drug Resist. 2020;13:761-71.

61. Kalil AC, Metersky ML, Klompas M, et al. Management of adults with hospital-acquired and ventilator-associated pneumonia: 2016 clinical practice guidelines by the Infectious Diseases Society of America and the American Thoracic Society. Clin Infect Dis. 2016;63(5):e61-111.

62. Torres A, Niederman MS, Chastre J, et al. International ERS/ ESICM/ESCMID/ALAT guidelines for the management of hospital-acquired pneumonia and ventilator-associated pneumonia: guidelines for the management of hospital-acquired pneumonia (HAP)/ventilator-associated pneumonia (VAP) of the European Respiratory Society (ERS), European Society of Intensive Care Medicine (ESICM), European Society of Clinical Microbiology and Infectious Diseases (ESCMID) and Asociacion Latinoamericana del Torax (ALAT). Eur Respir J. 2017;50(3):1700582.

63. Sartelli M, Chichom-Mefire A, Labricciosa FM, et al. The management of intra-abdominal infections from a global perspective: 2017 WSES guidelines for management of intra-abdominal infections. World J Emerg Surg. 2017;12:29.

64. Sartelli M, Weber DG, Ruppe E, et al. Antimicrobials: a global alliance for optimizing their rational use in intra-abdominal infections (AGORA). World J Emerg Surg. 2016;11:33.

65. European Association of Urology. EAU guidelines on urological infections. 2020. https://uroweb.org. Accessed 21 Dec 2020. 